\title{
Effect of HIV infection on the clinical response of leprosy in Northern Nigeria - a study done in $\mathbf{2 0 0 5}$
}

\section{Walter Kalu*}

Address: MBBS, Ebonyi State University, Abakaliki, Nigeria

* Corresponding author

from 2006 International Meeting of The Institute of Human Virology

Baltimore, USA. 17-21 November, 2006

Published: 21 December 2006

Retrovirology 2006, 3(Suppl I):P32 doi:I0.I I86/1742-4690-3-SI-P32

The clinical features associated with HIV in Adamawa State north eastern Nigeria was done clinically and bacteriologically using Ridley-Jopling classification and bacteriological index respectively. The cases were classified as paucibacillary (Tuberculoid - TT and Borderline Tuberculoid - BTT) and multibacillary (Borderline Borderline BB, Borderline Lepromatous - BL and Lepromatous Leprosy - LL) Leprosy.

Eleven (10\%) of 104 cases were HIV seropositive comprising 7 males and 4 female. Age range was 14 and 63 . Among the HIV seropositive patients, those with paucibacillary $(\mathrm{PB})$ were 6 (TT -1, BT -5) while multibacillary (MB) Leprosy 5 (BB-1), BL -2, LL-2). The predominant clinical feature were clawing fingers (64\%) ulceration $(64 \%)$, hand muscle atrophy $(5 \%)$ and clawing toes $(45 \%)$. Some clinical feature of paucibacillary leprosy such as sensory loss and hair losses as is also seen in HIV negative patients occurred in increase frequency in HIV positive patients belonging to the multibacillary class. The HIV infected leprosy patients are more likely to manifest advanced stages of the disease than HIV seronegative patient. 